\title{
X for Design: A Usable Framework for Enhancing Design Activities
}

www.jestr.org

\author{
Stefano Filippi* and Daniela Barattin
}

DPIA Department, University of Udine, 33100, Udine, Italy

Received 22 March 2018; Accepted 11 July 2019

\begin{abstract}
Current market requirements push designers towards the enhancement of their activities. One possibility for doing this consists in analyzing design activities from different points of view, aiming at identifying and implementing possible improvements. The literature already offers descriptive methods and tools to perform this analysis exhaustively and effectively; nevertheless, the increasing variety of starting points and goals as well as the exploitation of user-related concerns like emotions, meanings, etc., sometimes make these methods and tools barely suitable for this analysis. This research aims at developing the X for Design (XfD) framework to model design activities that deal with different starting points and goals as well as with user experience concerns, in order to enhance them by highlighting where to add something new and/or what to modify/delete of the existing. To achieve this, the analysis of existing design activities and design methods allows highlighting some requirements to overcome the weaknesses of the descriptive methods and tools currently available. Then, these requirements lead the development of the XfD framework, based on three well-known descriptive methods and tools. Subsequently, the adoption of the XfD to model and enhance the design activities of two real companies comes as first validation of the research results. This validation highlights both quantitative and qualitative improvements. The XfD could help researchers in deepening their knowledge about the role of emotions and human behaviors in design; at the same time, designers could adopt the framework to enhance their activities in order to match current market requirements at best.
\end{abstract}

Keywords: Design Activities, Human Behavior in Design, Modeling Methods and Tools, User Experience, $\mathrm{X}$ for Design

\section{Introduction}

Current market requirements demand multi-functional, innovative and user experience-oriented products. Time-tomarket is always shorter and R\&D resources are sometimes rather scant [1]. Design activities have already started evolving in order to satisfy these requirements. Several of them consider users' perceptions, sensations and emotions as key components for developing successful products [2]; these components belong to the user experience (UX), one of the domains that saw their importance increase in the recent years [3-4]. Moreover, design activities exploit heterogeneous starting points and goals to widen the chances to generate innovative products. Starting points (sources) can range from functions to shapes, materials, behaviors, etc.; goals (targets) can consist in other characteristics than product structures like shapes, functions, etc. There are many examples in the literature describing the characteristics of these design activities. Filippi and Barattin [5] describe design activities that start by considering generic shapes having a strong impact on users' emotions and generate product functions consequently. Particular shapes affect users' visual and aesthetic perceptions and suggest functions unconventional for specific types of products; these functions could increase both the innovation degree and the purchasing of products shaped that way [6-7]. In the research of Ashby and Johnson

*E-mail address: filippi@udine.it

ISSN: $1791-2377$ @ 2019 School of Science, IHU. All rights reserved.

doi:10.25103/iestr.124.23
[8], the analysis of different materials gives indications about the most appropriate shapes these materials can be associated with and about the perceptions, sensations and emotions a product made by these materials could arouse. Takada, Boer and Sawaragi [9] define the behavior of a product, a driver assist system, starting from users' needs and behaviors. Graziosi, Ferrise, Furtado and Bordegoni [10] describe the definition of product structures starting from users' perceptions about sounds, behaviors and visual appearance instead of from functions.

In order to satisfy current market requirements, designers can analyze their activities in detail, from different points of view, using descriptive methods and tools; this allows them identifying possible lacks and suggests possible improvements that they can implement to get their activities enhanced.

The literature already offers several methods and tools to describe design activities. For example, the situated Function-Behaviour-Structure framework [11] supports the description of the cognitive aspects of design activities that use functions as starting point. This description goes from the formulation of the technical specifications up to the definition and evaluation of the product structures. The model of March [12] describes design activities that begin with the productive reasoning drawing on a preliminary statement of required characteristics, aiming at producing the first design proposal; then, this proposal is deductively analyzed to predict the expected performance of the product; finally, further design possibilities are evaluated inductively. The Matchett's model [13] analyses design situations to identify the needs that the product should help 
to satisfy. Then, it classifies these needs into primary needs (those that must be satisfied) and secondary ones (eventually, these could be neglected); principles to satisfy the primary needs are searched and the set of design solutions able to satisfy both the primary and secondary needs is defined. Finally, a review of the outcomes evaluates both the functional effectiveness and the quality of the product according to materials and manufacturing processes. Cross [14] made a deep review of several engineering design activities and proposed a method composed by seven stages ranging from the clarification of the objectives and the functional analysis to the evaluation of the alternatives and improvement of details. Similar to this last method is that proposed by Pahl, Beitz, Feldhusen and Grote [15]; their method starts from the clarification of the task and the elaboration of the specifications and ends with the evaluation and documentation of the final solutions [16].

Unfortunately, these methods and tools are not fully suitable for describing and managing design activities involving UX concerns as well as a variety of starting points and goals altogether at best. For example, the FBS framework and the models of March and Matchett cannot model explicitly how users perceive the product and the emotions aroused meanwhile. At the same time, the method of Cross and that of Pahl et al. cannot describe design activities starting from shapes or materials (they establish that functional analysis is the second activity to perform after the clarification of the objectives to achieve and of the task to do); therefore, considering sources different from functions is not feasible. Moreover, these methods and tools can only describe designers' activities but they do not suggest how to enhance them.

This research aims at filling the gap by developing the framework named X for Design (XfD). The XfD will model design activities dealing with different sources and targets taking care of UX concerns meanwhile. Moreover, the XfD will enhance these activities by highlighting where to add something new and/or what to modify/delete of the existing. The "X for Design" name emphases the ability to describe design activities that deal with different sources/targets and UX concerns. This would be somehow in juxtaposition to the "Design for X" concept, representing the customization of design activities to generate products obeying to specific constraints (e.g., Design for Manufacturing, Assembly, Reliability, etc.) [17-19]. The XfD can be of help for different people involved in product development. Researchers could deepen their knowledge about the role of emotions and human behaviors in design; at the same time, designers could adopt the framework to enhance their activities in order to match current market requirements at best.

This paper runs as follows. The background section summarizes the starting points of the research: the FBS framework, the framework of the product experience, the model of the seven stages of action cycle and five reference design activities. The activity section starts by analyzing these reference design activities and design methods from literature. This analysis allows highlighting some requirements to overcome the weaknesses of the descriptive methods and tools currently available. The descriptions of the XfD architecture and tools take place afterwards. Then, the next section reports the first validation of the XfD in the field thanks to its adoption to enhance the design activities of two real companies. The discussion about the research results, together with some conclusions and hints for future work, close the paper.

\section{Background}

\subsection{The situated FBS framework with extension}

The Function-Behaviour-Structure framework is a conceptual scheme that generalizes and organizes heterogeneous groups of entities and processes to begin and continue design activities [20-22]. It is an ontology made by classes of variables and by their transformations during the activities of a generic design process [23-24]. This framework has been updated more times and this research considers the most recent release, the situated FBS framework [11] with the extension described in Cascini, Fantoni and Montagna [25]. This release, called for brevity FBS from now on, includes important elements as the definition of different environments (worlds) and the possibility to consider further actors than designers (all of them are called agents). Moreover, particular attention is placed on user needs and product requirements.

The FBS models "function-based" design activities, those activities that generate technical specifications starting from the user needs that the product should help to satisfy. This modeling happens thanks to five variables, managed in three different worlds by ten reference processes composed of lists of sub-processes. Fig. 1 represents all of this graphically.

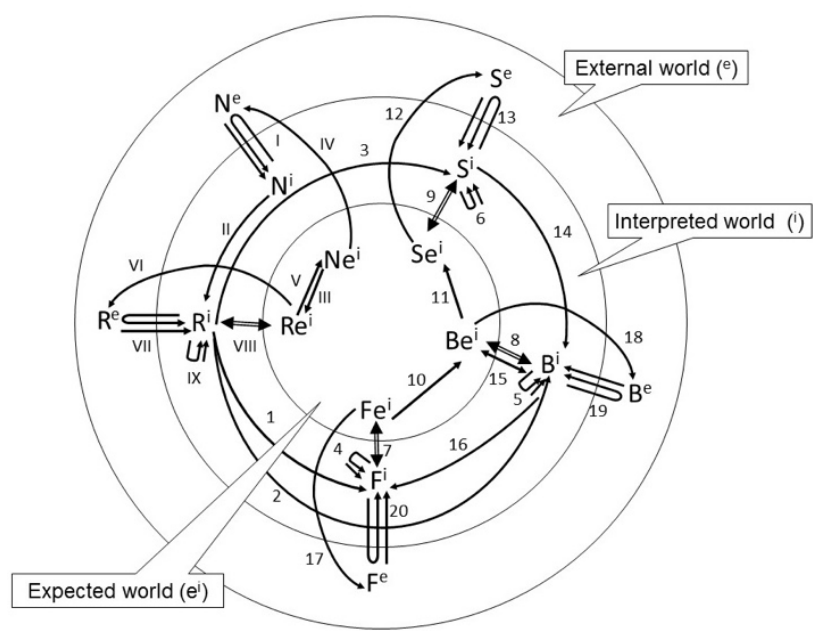

Fig. 1. The FBS.

The five variables are as follows.

- Need (N). It expresses perceived desirable situations to be attained or undesirable situations to be avoided, explicitly stated by the designers or perceived by observing real users.

- Requirement (R). It represents measurable properties of the product related to one or more needs.

- Function (F). It describes the aim of the product, i.e., what the product is for.

- Structure (S). It describes product components in terms of shapes, dimensions and materials, and their relationships, i.e., what the product is.

- Behavior (B). It describes the attributes derived or expected from the structures of the product, i.e., what the product does. 
The three worlds where the variables are managed refer to the designers' perceptive sphere. These worlds are as follows.

- External world $\left({ }^{\mathrm{e}}\right)$. It is composed of real representations outside the designers' mind.

- Interpreted world $\left({ }^{\mathrm{i}}\right)$. It is built up inside the designers' mind in terms of sensory experiences, percepts and concepts. It is the internal, interpreted representations of that part of the external world the designers interact with.

- Expected world $\left(\mathrm{e}^{\mathrm{i}}\right)$. It is the world where the effects of the designers' actions are imagined according to the goals and the interpretations of the current state of the external world.

These worlds are put into relationship to each other by exploiting four different classes of sub-processes. These classes are as follows.

- Push-pull ( $\subseteq$ ). The sub-processes of this class implement the interaction of an agent with its external environment (the external world) as well as with its internal environment (the interpreted world).

- Focussing $(\Leftrightarrow)$. The sub-processes belonging to this class point out some aspects of the interpreted world and these aspects are used as goals in the expected world.

- Action $(\rightarrow)$. The sub-processes of this class generate an effect, which brings about a change in the external world according to the goals in the expected world.

- Comparison $(\leftrightarrow)$. The sub-processes of this class compare the expected $\mathrm{N}, \mathrm{R}, \mathrm{F}, \mathrm{S}$ and $\mathrm{B}$, with the interpreted ones.

The ten reference processes that model the design activities "function-based" are as follows.

- Need identification. Here the customer needs $\mathrm{N}^{\mathrm{e}}$ are investigated and transformed into expected needs $\mathrm{Ne}^{\mathrm{i}}$, the goals to achieve thanks to the design activities.

- Requirement definition. It analyses and interprets the expected needs $\mathrm{Ne}^{\mathrm{i}}$ to generate the requirements $\mathrm{R}^{\mathrm{i}}$, the basis for the generation of the design solutions.

- Formulation. This process produces the interpreted representations of the $\mathrm{F}, \mathrm{B}$ and $\mathrm{S}$ variables $\left(\mathrm{F}^{\mathrm{i}}, \mathrm{B}^{\mathrm{i}}\right.$ and $\mathrm{S}^{\mathrm{i})}$ starting from the interpreted requirements; then, it focuses on the initial design state space constituted by their expected representations $\mathrm{Fe}^{\mathrm{i}}, \mathrm{Be}^{\mathrm{i}}$ and $\mathrm{Se}^{\mathrm{i}}$.

- Synthesis. It generates the external representation of the object structure $\mathrm{S}^{\mathrm{e}}$, starting from its expected behavior $\mathrm{Be}^{\mathrm{i}}$.

- Analysis. It investigates the synthesized object structure $\mathrm{S}^{\mathrm{e}}$ to gather the interpreted behavior $\mathrm{B}^{\mathrm{i}}$.

- Evaluation. It compares the interpreted behavior $\mathrm{B}^{\mathrm{i}}$ to the expected behavior $\mathrm{Be}^{\mathrm{i}}$ thanks to the analysis of the design solutions.

- Documentation. This process generates the description of the design solutions (e.g. for manufacturing, assembly, etc.) after the achievement of a positive evaluation of them.

- Reformulation (types 1,2 and 3). If the solutions should not be satisfactory (they appear unable to solve the design problem in full), the reformulation processes reapply some sub-processes belonging to the previous processes to address corrections and improvements.
The three types correspond to the variable the solutions refer to: F, B and S, respectively.

These processes are implemented using thirty subprocesses. Their description is not present here for space reasons; nevertheless, almost all of them compare in the rest of the paper. An example of sub-process is proposed to clarify their notation and meaning. The first sub-process of the need identification is represented by $\mathrm{N}^{\mathrm{e}} \subseteq \mathrm{N}^{\mathrm{i}}$ (labelled as "I" in fig. 1). The meaning of this notation is that the external needs $\left(\mathrm{N}^{\mathrm{e}}\right)$ are translated $(\subseteq)$ into interpreted ones $\left(\mathrm{N}^{\mathrm{i}}\right)$ (by the designers thanks to their own skill and knowledge).

This research involves the FBS because its architecture (variables, worlds and classes of sub-processes) offers a high level of organization and precision in the description of design activities as well as of that flexibility required to manage different sources, targets and to allow activity modifications.

\subsection{The framework of the product experience}

Winckler, Bernhaupt and Bach [4], Hassenzahl and Tractinsky [26] and Jordan [27] offer a general definition and a classification of UX components. Examples of UX components are simulation, emotions, social relatedness/coexperience, meanings and identification. The framework of the product experience [3] analyses the cognitive activities involved in different types of affective users' experiences with products and describes the relationships among these activities. The product experience is the change in core affect attributed to user-product interaction. It is a multifaceted phenomenon composed of three UX components: the aesthetic experience, experience of meaning, and emotional experience. The aesthetic experience represents the ability of a product to delight one or more human sensorial modalities, i.e., it describes how the users perceive the product through their senses. The experience of meaning allows the personal or symbolic significance of a product to be set. Thanks to this experience, people recognize metaphors and highlight expressive product characteristics; consequently, precise meanings are assigned to the product. Individual and cultural differences influence this experience. The emotional experience represents the interpretation of a product based on events considered as potentially beneficial or harmful. Users' personality and past experiences influence this experience. The three components are conceptually separated; nevertheless, it is difficult to distinguish them in an everyday product experience because the emotional experience is directly influenced by the others; emotions come from the evaluation of events sensed through the aesthetic experience or derived from the experience of meaning.

The product experience framework is important for this research because it allows representing and managing UX concerns and especially users' perceptual and emotional matters in design.

\subsection{The model of the seven stages of action cycle}

The model of the seven stages of action cycle proposed by Norman [28] is an approximated model of human cognition and behavior. It is composed of three levels - hierarchically ordered against the consciousness degree in the human mind - that work together. The first level is the visceral level, unconscious, that expresses the basic protective mechanisms of the human affective system by making quick judgments about perceived stimuli and generating automatic actions as answers to these stimuli. The second level is the behavioral 
one, partially conscious, that represents the home of the behavioral skills triggered by situations that match specific patterns. The third level is the reflective level, conscious, where reasoning and decision-making processes occur in a deep and slow way; these processes often occur when events have already happened. The seven stages of action take place in these three levels as follows. As soon as a stimulus comes from the external world, the human being perceives (I stage) it through the senses at the visceral level. Then, the stimulus is interpreted (II) and becomes internal information at the behavioral level. Now, the human being compares (III and IV) this information with the goals established before starting the interaction at the reflective level. The results of the comparison allow the human being to plan (V) the next action to do (always at the reflective level), to specify (VI) his/her behavior (at the behavioral level) and to perform (VII) the action, before in his/her mind (at the visceral level) and in the external world afterwards.

This research needs the contribution of the seven stages of action cycle model to make the XfD able to define and manage user behaviors and cognitive mechanisms.

\subsection{Reference design activities}

The research described in this paper uses five different design activities. They are considered as references; their description appears in the following.

\subsubsection{From functions to structures (RDA1)}

The design of CNC (Computer Numerical Control) milling machines can be considered a good example of classic design activities following the paradigm "from functions to structures" [29]. Functions come directly from the users. These functions are analyzed and transformed into behaviors to understand how the machines must work due to specific requirements and constraints (e.g., dimensional precision, manufacturing processes, materials, etc.). Then, these behaviors are transformed into structures and prototypes are built. These prototypes allow checking and validate the design outcomes and help highlighting possible improvements [30]. These reference design activities are the first ones considered in this research; thus, they are labelled as RDA1.

\subsubsection{From shapes to functions (RDA2)}

An example of "from shapes to functions" design paradigm comes from Alessi, an iconic Italian company producing home appliances. This company asks architects and graphical designers, expert of fashion trends, for generating shapes that should arouse particular emotions in the users [31-32]. Alessi's design activities start with tests where users see and touch shapes and express functions they expect from products shaped that way. In this case, functions are the design goals. Once expressed by the testers, these functions are compared with those highlighted by designers thanks to the analysis of the needs. This comparison supports the definition of the ultimate design goals and prototypes are built on them. The prototype evaluation leads the final refinement of the design outcomes.

\subsubsection{From sounds, behaviors and shapes to structures (RDA3)}

The optimization of the structure of a dishwasher door is an example of these design activities [10]. Designers invite users to consider the sounds, behavior and visual appearance (shapes and dimensions) of the door while opening and closing it; these sources are represented/generated by exploiting a mixed reality prototype [33]. Then, the users imagine and describe the characteristics the door should have by considering the perceived ones. This feedback allows the designers to modify the structure of the door to match the users' desiderata. After the modifications, tests start over if required.

\subsubsection{From materials to shapes (RDA4)}

As reported by Ashby and Johnson [8], the shapes of chairs, buildings, etc., are strongly conditioned by the characteristics of the materials they are made of. This would suggest "from materials to shapes" as one of the design activities to consider here. Considerations about the physical characteristics of materials allow understanding the behaviors of products made by those materials due to specific conditions (e.g., deformations of chair seats built using a specific foam rubber due to different people's weights or different ways to seat on it; possible oscillations of buildings made by specific kinds of concrete due to the wind force, etc.). Then, designers consider materials looking at and touching them to collect emotions during interaction. The outcomes of the two analyses allow generating those product shapes that guarantee the architectural standards and arouse specific emotions at the same time.

\subsubsection{From needs to product behaviors (RDA5)}

Takada, Boer and Sawaragi [9] develop the Advanced Driver Assistance Systems (ADAS) starting from the analysis of the needs (reducing risks and efforts, maintaining contextual awareness, achieving a satisfactory level of performance, etc.) and aiming at achieving the system behaviors that satisfy drivers as best as possible. This design approach would suggest considering here the "from needs to product behaviors" design activities. Thanks to the identification and analysis of the needs involving drivers (future users of the system), designers define the functions of that system. In turn, these functions allow highlighting the product behaviors the drivers would like to get available. The ADAS behaviors are defined in order to make all of this possible.

\section{Activities}

The research starts by defining the requirements for the development of the XfD framework in order to overcome the weaknesses of the existing methods and tools; this activity occurs by analyzing the reference design activities described in section 2.4 and by exploiting existing design methods. After that, the XfD development takes place, followed by its adoption in enhancing the reference design activities considered at the beginning.

\subsection{Definition of the requirements for the $\mathrm{XfD}$ development}

In order to define the general requirements for the $\mathrm{XfD}$ development, five reference design activities and four methods focusing on design processes are involved. The design activities are those described in section 2.4; they come from direct observations in the field or from the literature. Their selection aims at exploring different contexts, having different starting points and goals, involving users and considering UX concerns. The four design methods come from the literature; they are very known and used, they involve different design activities and tools and are suitable for different products and contexts. These methods are the design method of Cross [14], the 
systematic approach of design engineering proposed by Pahl et al. [15], the mechanical design process of Ullman [34] and the Norman's design for UX [28]. The result of this analysis consists of the following requirements.

- First requirement (REQ1): the XfD must use a unique set of sources/targets. The reference activities highlight the multiplicity of the elements they start from (sources), the product characteristics they aim developing at (targets), as well as the kind of data elaborations to get the latter from the former. The sources refer to physical characteristics like shapes (reference design activities RDA2 and RDA3), sounds (RDA3) and materials (RDA4), as well as to product functions (RDA1), product behaviors (RDA3) and needs (RDA5). Indeed, it is straightforward to note that the targets show the same characteristics; they can refer to physical characteristics of the product like structures (RDA1 and RDA3) and shapes (RDA4), as well as to product functions (RDA2) and product behaviors (RDA5). Therefore, sources and targets are somehow the same thing and this allows them to be collected using a unique set. Thanks to this, possible information redundancy and/or misalignment disappear; at the same time, this supports some simplifications in the XfD architecture.

- Second requirement (REQ2): the XfD must address users explicitly and model the UX concerns. The description of RDA2, RDA4 and RDA5, as well as the Norman's design for UX highlight the role and importance of users in design, mainly from the emotional matters point of view. Aroused emotions (RDA2 and RDA4) can lead to suggestions and ideas to develop into new products to attract future users, while the analysis of human behaviors (RDA5) can help in defining the product behaviors to support the users as best as possible.

-

- Third requirement (REQ3): the XfD must have a flexible and updatable architecture. The existing design methods considered here, together with the variety of the reference design activities, suggest an XfD architecture as flexible and updatable as possible to allow introducing new sources and targets as well as new activities in existing models easily. For example, design activities could change over time because of a required analysis of an unexpected source of information. The XfD should be flexible enough to allow updating the existing model of these design activities without restarting the modeling from the beginning.

-

- Fourth requirement (REQ4): the XfD must be usable. The methods of Ullman and that of Norman made us get in contact with people belonging to different contexts, showing different skills, knowledge and experience. Since the XfD would like to be of help to all of them as much as possible, its adoption must be very easy.

\section{2. $\mathrm{XfD}$ architecture}

\subsubsection{Synergy among FBS, framework of the product experience and model of the seven stages of action cycle} Existing descriptive methods and tools can satisfy these four requirements singularly but none is able to answer to them altogether. For example, the FBS satisfies REQ3 because its architecture is flexible and easily updatable, but it does not satisfy REQ2 because it cannot model emotions. On the contrary, the framework of the product experience satisfies
REQ2 because it can describe the experience that the product arouses in the user by focusing on aesthetics, meanings and emotions but it does not satisfy REQ1 because it cannot manage variables explicitly. Thus, the effort here is to make different descriptive methods and tools work in synergy in order to exploit their specific abilities in satisfying each requirement. The core of the XfD copies the FBS architecture; specifically, the FBS components exploited here are the variables, the worlds and the classes of sub-processes (the building blocks for modeling the design activities). This helps fulfilling both REQ1 - since variables are managed explicitly, easily and in a uniform way, and REQ3 - given that the generic definition of the FBS components shows the required flexibility and updatability to allow changes. Along with the FBS, the framework of the product experience gives its contribution to the XfD in order to make it able to model the consideration about the users. Three elements of the framework of the product experience are exploited: the three components (the aesthetic experience, experience of meaning and emotional experience), the relationships among them and the conditions that influence them (individual and cultural differences, users' personality and past experiences). These three elements are of great help in representing and managing users' perceptual, behavioral and emotional matters in design; therefore, all of them help in fulfilling REQ2. The model of the seven stages of action cycle is exploited here to satisfy REQ2 as well. It allows making the cognitive mechanisms explicit by explaining how the human mind works in generating a reaction starting from a perceived stimulus. It is worth to say that when comparing the FBS and the model of the seven stages of action cycle, both of them start from the external world, collect and cognitively elaborate pieces of information to achieve a precise goal, and express this goal again outside the cognitive sphere; apparently, these two tools describe the same thing. The difference consists in the point of view. The FBS emphasizes variables, worlds and sub-processes, and considers them as atomic entities, building blocks, without going inside them further; on the contrary, the model of the seven stages of action cycle focuses on processing, on how the elaboration of the pieces of information takes place. The structure of the three levels - visceral, behavioral and reflective, as well as the seven stages, their meaning and relationships, will be of great help in covering the lacks of consideration about the "how" the human reasoning and behavior take place and about the "how" the emotions influence both of them. Finally, the satisfaction of REQ4, the requirement referring to usability matters, needs the FBS, the framework of the product experience and the model of the seven stages of action cycle altogether; the way this happens will be made clear in the following.

\subsubsection{XfD variables}

The XfD uses the FBS variables as they are. This because they seem to be able to represent all kinds of sources/targets present now in the set. For example, the definition of the FBS variable $\mathrm{S}$ in terms of shape, dimensions, material and relationships among them, covers the whole set of physical characteristics of products (e.g. shapes, materials, sounds, etc.). Nevertheless, three variables need to be added to describe the product experience at best; they are aesthetics (A), meaning (M) and emotion (E). They do not belong to the sources/targets set; instead, they are internal variables. Finally, one more variable is required to consider the users in the design activities. This variable is human behavior 
$(\mathrm{hB})$; it describes the behavior of those who will interact with the product under development. The FBS variable B describes product behavior; no FBS variables take care of human behavior.

\subsubsection{XfD environments}

The environments in which the XfD variables are considered are the same as in the FBS; therefore, the three worlds (external, interpreted and expected) are present here, along with their relationships and with the same rules to move within the same world and between different worlds.

\subsubsection{XfD processes}

As for the FBS architecture, the XfD translates design activities into processes. Each process consists of subprocesses that describe single design activities by exploiting the same classes of sub-processes of the FBS, except for a little add-on. The push-pull class and, in particular, the pull part of it, in addition to identifying sub-processes allowing a further interpretation of a variable without any influence, is meant here as the class of those sub-processes where the interpretation of a variable can be influenced by a different one. For example, consider the sub-process $\mathrm{A}^{\mathrm{i}} \subseteq \mathrm{E}^{\mathrm{i}}$ involving aesthetics and emotion variables, two out of the four new variables introduced during the development of the XfD. The meaning of this sub-process is that the interpreted aesthetics $\left(\mathrm{A}^{\mathrm{i}}\right)$, achieved thanks to the perception of a specific stimulus, influence $(\subseteq)$ the interpreted emotions $\left(E^{i}\right)$ that will be used in later sub-processes.

\subsection{XfD tools}

The XfD contains two main tools, the IF-THE conditional statements and the rules, to model and refine design activities, respectively. These tools aim at making the XfD suitable for researchers and/or designers with different skills and expertise. In turn, this fulfil REQ4, referring to usability. Fig. 2 outlines the role of these tools in the XfD adoption; the description of them is in the following.

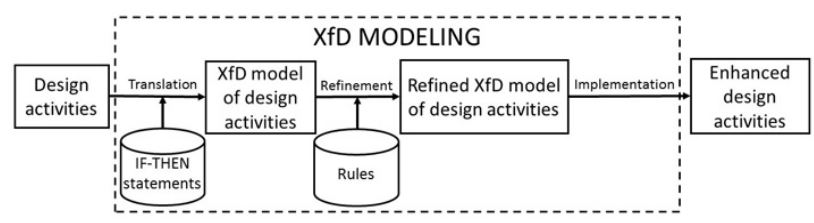

Fig. 2. Outline of the XfD adoption.

\subsubsection{IF-THEN conditional statements}

An ordered list of eleven IF-THEN conditional statements allows translating design activities into sequences of subprocesses, the language used by the XfD. These statements come from the FBS and refer to generic activities. The statements appear as ordered temporally to help in applying them correctly, without requiring specific knowledge about the relationships between worlds and classes of subprocesses, etc. To apply the statements, the XfD users researchers and/or designers, start by identifying each activity to model. Then, they select the statements considering those ones showing the most similar generic description (the IF part of the statement) to that activity. All suitable statements must be considered, in the order they appear in the list. After that, the XfD users contextualize these statements to highlight the proper variables and translate the activity into the corresponding sub-process (or sequence of sub-processes). At the end, the resulting ordered list of sub-processes represents the model of the design activities as they are. For example, consider the development of loud alarms. The first design activity is "analysis of different sound types"; the designers of the company analyze and elaborate a set of sounds that could be used as alarms. The source of this activity is the sound; the $S$ variable represents it. This activity implies an analysis and this leads to the selection of the statements "IF the design activity deals with the analysis of a specific variable and this variable has never been experienced by the designers (it has never been interpreted in the designers' mind), THEN this variable must be interpreted using the push-pull class (to be precise, here the push is involved)" and "IF the design activity deals with the analysis of a specific variable and this variable has been experienced by the designers (interpreted in the designers' mind), THEN this variable must be further interpreted by exploiting similar designers' past experiences (previous knowledge) using the push-pull class (to be precise, here the pull is involved)". The contextualization of the first statement respect to the sound variable (source) suggests the interpretation of the specific set of sounds in the designers' mind (from the external to the interpreted world); this is translated into the sub-process $\mathrm{S}^{\mathrm{e}} \subseteq \mathrm{S}^{\mathrm{i}}$. Then, these interpreted sounds are further elaborated by exploiting the previous knowledge of the designers about similar sounds belonging to different products; the second statement translates it into the sub-process $\mathrm{S}^{\mathrm{i}} \subseteq$. These sub-processes are then introduced in the model according to the order of the statements they come from.

\subsubsection{Rules}

Once the model is generated using the statements, the XfD provides a set of twelve rules to refine it in order to enhance the design activities. This refinement happens by highlighting those sub-processes that the exploitation of the statements could have missed. Such omissions come mainly from the XfD users' underestimation or unawareness of the corresponding activities in the real product development. Rules may suggest introducing new sub-processes dealing with different variables and/or belonging to different classes of sub-processes. These rules come from all the descriptive methods and tools considered in the research (FBS, framework of the product experience and model of the seven stages of action cycle) and they are generic; they do not refer to any specific design context or situation. The XfD users select specific rules depending on the variables and subprocesses involved time by time and generate the missing sub-processes accordingly. For example, consider again the development of loud alarms. During the prototype generation, the technical specifications of the prototype (expected product structures) are selected from the list of concepts defined by the designers (interpreted structures); the statements translated this selection into the sub-process $\mathrm{S}^{\mathrm{i}} \Leftrightarrow \mathrm{Se}^{\mathrm{i}}$. The rule "expected structures should be achieved by focussing on interpreted structures and by transforming expected behaviors", suggested by the formulation and synthesis processes of the FBS, highlights a gap because, by applying the statements only, the expected product structures would be selected considering only the focussing, without involving any transformation. Therefore, the rule suggests adding the sub-process $\mathrm{Be}^{\mathrm{i}} \rightarrow \mathrm{Se}^{\mathrm{i}}$ to the model. The introduction of the sub-processes suggested by the rules enriches the model; their implementation back into design activities by the XfD users enhance them. 


\section{First validation of the XfD}

This section describes the first two adoptions of the XfD in the field. They start demonstrating the value of the XfD in enhancing design activities and its usability. These adoptions concern two companies involving different products, sources, targets and design activities. Moreover, both of them deal with UX concerns. Designers of these companies use the XfD to model their specific activities. Two XfD developers follow the adoptions and help designers when blocking problems arise. For example, they intervene if designers do not fully understand the meaning of some rule or cannot apply statements and rules in particularly difficult situations. After the generation of the two models, all the designers fill a first questionnaire composed by four open questions focused on the usability of the XfD tools. For example, designers are asked if the language used by statements and rules is comprehensible, if it is clear when to apply the rules and where to add the refined sub-processes, etc. To compute the amount of the $\mathrm{XfD}$ enhancement, designers are divided into two groups. One group performs the original activities (those the companies are used to) and the other group performs the enhanced ones. All of them design/redesign the same specific product in each company. At the end, the group of designers who performed the enhanced design activities fills a second questionnaire composed by five open questions evaluating the execution of these activities in terms of enhancement/worsening of activities, design outcomes, time spent to carry on the activities, etc. The XfD developers collect the results (the models, the design outcomes achieved with both the original and enhanced design activities and the questionnaires) and compare them. Clearly, this is not a conclusive validation of the XfD because further experiences in the field as well as comparisons of the design outcomes to those obtained by involving different methods and tools are required. All of this is in progress and the results will be published in the future.

\subsection{First $\mathrm{XfD}$ adoption in the field}

The first adoption in the field involves a company producing pieces of furniture; it has one plant only but it sells products in many countries thanks to e-commerce facilities. The company focuses its interests on user experience matters since it aims at generating products that follow current fashion trends arousing specific emotions. The design activities considered here runs from the analysis of shapes to the production of pieces of furniture. For space reasons, only the first four phases are modeled, up to the generation of the first prototype. The first phase is the analysis of the shapes, from which a preliminary collection of product functions and behaviors is derived. Functions and behaviors are then further analyzed to define the technical specifications (expressed in terms of product structures) that represent the design goals; this is the second phase. The third phase deals with the evaluation of the feasibility of the goals due to manufacturing processes as well as respect to current safety regulations about materials, ergonomics and users' health in general. After that, the generation of the first prototype takes place in the fourth phase.

Six designers of furniture for kitchens (e.g., tables and chairs, sideboards, etc.) were involved and generated the model of their activities using the XfD. Left side of tab. 1 reports the result. The generation of the full model (translation and refinement) took one hour and twenty-five minutes; designers spent thirty-five minutes in applying the statements, twenty-five minutes for the rules and twenty-five minutes for the implementation of the refined sub-processes. Designers needed two times the help of the XfD developers in applying the rules. This happened in the first phase, for introducing the meaning variable and the sub-processes that relate it with shapes, aesthetics, emotions and human behaviors. This help was necessary because designers did not know the role of this variable and how to apply it. Up to that moment, they had considered emotions only. In the second and fourth phases, the XfD developers intervened to help introducing the relationships between the expected behaviors and the expected structures. This happened because, once defined the structures, it was unusual for the designers to go back considering product behaviors to define the final expected structures.

Once generated the model, all the designers filled the first questionnaire. After that, they were divided in two groups; three of them performed the original activities and the other three the enhanced ones, all of them aiming at designing a new kitchen chair. At the end, the designers that performed the enhanced activities filled the second questionnaire.

Table 1. Models of the design activities in the first two XfD adoptions in the field.

\begin{tabular}{|c|c|c|c|c|}
\hline & \multicolumn{2}{|c|}{ First adoption (chair design) } & \multicolumn{2}{|c|}{ Second adoption (washbasin redesign) } \\
\hline & Phase & $\begin{array}{l}\text { Translation/ } \\
\text { Refinement }\end{array}$ & Phase & $\begin{array}{l}\text { Translation/ } \\
\text { Refinement }\end{array}$ \\
\hline 1 & Shape analysis & $\mathrm{S}^{\mathrm{e}} \subseteq \mathrm{S}^{\mathrm{i}}$ & Definition of expected user & $\mathrm{F}^{\mathrm{e}} \subseteq \mathrm{F}^{\mathrm{i}}$ \\
\hline 2 & & $\mathrm{~S}^{\mathrm{i}} \subseteq$ & and product behaviours & $\mathrm{F}^{\mathrm{i}} \subseteq$ \\
\hline 3 & & $\mathrm{~S}^{\mathrm{i} \rightarrow} \rightarrow \mathrm{A}^{\mathrm{i}}$ & & $\mathrm{F}^{\mathrm{i}} \rightarrow \mathrm{M}^{\mathrm{i}}$ \\
\hline 4 & & $\mathrm{~A}^{\mathrm{i}} \subseteq$ & & $\mathrm{M}^{\mathrm{i}} \subseteq$ \\
\hline 5 & & $\mathrm{~S}^{\mathrm{i}} \rightarrow \mathrm{M}^{\mathrm{i}}$ & & $\mathrm{F}^{\mathrm{i}} \rightarrow \mathrm{E}^{\mathrm{i}}$ \\
\hline 6 & & $\mathrm{~A}^{\mathrm{i}} \subseteq \mathrm{M}^{\mathrm{i}}$ & & $\mathrm{M}^{\mathrm{i}} \subseteq \mathrm{E}^{\mathrm{i}}$ \\
\hline 7 & & $\mathrm{M}^{\mathrm{i}} \subseteq$ & & $\mathrm{E}^{\mathrm{i}} \subseteq$ \\
\hline 8 & & $\mathrm{~S}^{\mathrm{i}} \rightarrow \mathrm{E}^{\mathrm{i}}$ & & $\mathrm{M}^{\mathrm{i}} \Leftrightarrow \mathrm{Me}^{\mathrm{i}}$ \\
\hline 9 & & $\mathrm{~A}^{\mathrm{i}} \subseteq \mathrm{E}^{\mathrm{i}}$ & & $\mathrm{E}^{\mathrm{i}} \Leftrightarrow \mathrm{Ee}^{\mathrm{i}}$ \\
\hline 10 & & $\mathrm{M}^{\mathrm{i}} \subseteq \mathrm{E}^{\mathrm{i}}$ & & $\mathrm{Me}^{\mathrm{i}} \rightarrow \mathrm{hBe}^{\mathrm{i}}$ \\
\hline 11 & & $\mathrm{E}^{\mathrm{i}} \subseteq$ & & $\mathrm{Ee}^{\mathrm{i}} \rightarrow \mathrm{hBe}^{\mathrm{i}}$ \\
\hline 12 & & $\mathrm{M}^{\mathrm{i}} \Leftrightarrow \mathrm{Me}^{\mathrm{i}}$ & & $\mathrm{hBe}^{\mathrm{i}} \rightarrow \mathrm{hB}^{\mathrm{e}}$ \\
\hline 13 & & $\mathrm{E}^{\mathrm{i}} \Leftrightarrow \mathrm{Ee}^{\mathrm{i}}$ & & $\mathrm{hB}^{\mathrm{e}} \subseteq \mathrm{hB}^{\mathrm{i}}$ \\
\hline 14 & & $\mathrm{Me}^{\mathrm{i}} \rightarrow \mathrm{hBe}^{\mathrm{i}}$ & & $\mathrm{hB}^{\mathrm{i}} \subseteq$ \\
\hline
\end{tabular}




\begin{tabular}{|c|c|c|c|c|}
\hline $\begin{array}{l}15 \\
16 \\
17 \\
18\end{array}$ & & $\begin{array}{l}\mathrm{Ee}^{\mathrm{i}} \rightarrow \mathrm{hBe}^{\mathrm{i}} \\
\mathrm{hBe}^{\mathrm{i}} \rightarrow \mathrm{hB}^{\mathrm{e}} \\
\mathrm{hB}^{\mathrm{e}} \subseteq \mathrm{hB}^{\mathrm{i}} \\
\mathrm{hB}^{\mathrm{i}} \subseteq\end{array}$ & & $\begin{array}{l}\mathrm{hB}^{\mathrm{i}} \rightarrow \mathrm{B}^{\mathrm{i}} \\
\mathrm{B}^{\mathrm{i}} \subseteq \\
\mathrm{B}^{\mathrm{i}} \Leftrightarrow \mathrm{Be}^{\mathrm{i}} \\
\mathrm{Be}^{\mathrm{i}} \rightarrow \mathrm{B}^{\mathrm{e}}\end{array}$ \\
\hline 19 & & $\mathrm{hB}^{\mathrm{i}} \rightarrow \mathrm{B}^{\mathrm{i}}$ & \multirow{22}{*}{$\begin{array}{l}\text { Highlighting of real user } \\
\text { and product behaviours }\end{array}$} & $\mathrm{S}^{\mathrm{e}} \subseteq \mathrm{S}^{\mathrm{i}}$ \\
\hline 20 & & $\mathrm{~B}^{\mathrm{i}} \rightarrow \mathrm{F}^{\mathrm{i}}$ & & $\mathrm{S}^{\mathrm{i}} \subseteq$ \\
\hline 21 & & $\mathrm{~B}^{\mathrm{i}} \subseteq$ & & $\mathrm{S}^{\mathrm{i}} \rightarrow \mathrm{B}^{\mathrm{i}}$ \\
\hline 22 & & $\mathrm{~B}^{\mathrm{i}} \Leftrightarrow \mathrm{Be}^{\mathrm{i}}$ & & $\mathrm{B}^{\mathrm{i}} \subseteq$ \\
\hline 23 & & $\mathrm{~F}^{\mathrm{i}} \subseteq$ & & $\mathrm{S}^{\mathrm{i} \rightarrow \mathrm{A}^{\mathrm{i}}}$ \\
\hline 24 & \multirow[t]{24}{*}{ Goal definition } & \multirow{19}{*}{$\begin{array}{l}\mathrm{F}^{\mathrm{i}} \Leftrightarrow \mathrm{Fe}^{\mathrm{i}} \\
\quad \mathrm{Be}^{\mathrm{i}} \rightarrow \mathrm{Fe}^{\mathrm{i}} \\
\mathrm{Fe}^{\mathrm{i}} \rightarrow \mathrm{F}^{\mathrm{e}} \\
\mathrm{F}^{\mathrm{e}} \subseteq \mathrm{F}^{\mathrm{i}} \\
\mathrm{F}^{\mathrm{i}} \subseteq \\
\mathrm{F}^{\mathrm{i}} \rightarrow \mathrm{M}^{\mathrm{i}} \\
\mathrm{F}^{\mathrm{i}} \rightarrow \mathrm{E}^{\mathrm{i}} \\
\quad \mathrm{M}^{\mathrm{i}} \subseteq \\
\mathrm{M}^{\mathrm{i}} \subseteq \mathrm{E}^{\mathrm{i}} \\
\mathrm{E}^{\mathrm{i}} \subseteq \\
\mathrm{M}^{\mathrm{i}} \Leftrightarrow \mathrm{Me}^{\mathrm{i}} \\
\mathrm{E}^{\mathrm{i}} \Leftrightarrow \mathrm{Ee}^{\mathrm{i}} \\
\mathrm{Me}^{\mathrm{i}} \rightarrow \mathrm{hBe}^{\mathrm{i}} \\
\mathrm{Ee}^{\mathrm{i}} \rightarrow \mathrm{hBe}^{\mathrm{i}} \\
\mathrm{hBe}^{\mathrm{i}} \rightarrow \mathrm{hB}^{\mathrm{e}} \\
\mathrm{hB}^{\mathrm{e}} \subseteq \mathrm{hB}^{\mathrm{i}} \\
\mathrm{hB}^{\mathrm{i}} \subseteq \\
\mathrm{hB}^{\mathrm{i}} \rightarrow \mathrm{B}^{\mathrm{i}} \\
\mathrm{B}^{\mathrm{i}} \subseteq\end{array}$} & & $\mathrm{A}^{\mathrm{i}} \subseteq$ \\
\hline 25 & & & & $\mathrm{~S}^{\mathrm{i}} \rightarrow \mathrm{M}^{\mathrm{i}}$ \\
\hline 26 & & & & $\mathrm{~A}^{\mathrm{i} \subseteq \mathrm{M}^{\mathrm{i}}}$ \\
\hline 27 & & & & $\mathrm{M}^{\overline{\mathrm{i}} \subseteq}$ \\
\hline 28 & & & & $\mathrm{~S}^{\mathrm{i}} \rightarrow \mathrm{E}^{\mathrm{i}}$ \\
\hline 29 & & & & $\mathrm{~A}^{\mathrm{i}} \subseteq \mathrm{E}^{\mathrm{i}}$ \\
\hline 30 & & & & $\mathrm{M}^{\mathrm{i}} \subseteq \mathrm{E}^{\mathrm{i}}$ \\
\hline 31 & & & & \\
\hline 32 & & & & $\mathrm{M}^{\mathrm{i}} \Leftrightarrow \mathrm{Me}^{\mathrm{i}}$ \\
\hline 33 & & & & $\mathrm{E}^{\mathrm{i}} \Leftrightarrow \mathrm{Ee}^{\mathrm{i}}$ \\
\hline 34 & & & & $\mathrm{Me}^{\mathrm{i}} \rightarrow \mathrm{hBe}^{\mathrm{i}}$ \\
\hline 35 & & & & $\mathrm{Ee}^{\mathrm{i}} \rightarrow \mathrm{hBe}^{\mathrm{i}}$ \\
\hline 36 & & & & $\mathrm{hBe}^{\mathrm{i}} \rightarrow \mathrm{hB}^{\mathrm{e}}$ \\
\hline 37 & & & & $\mathrm{hB}^{\mathrm{e}} \subseteq \mathrm{hB}^{\mathrm{i}}$ \\
\hline 38 & & & & $\mathrm{hB}^{\mathrm{i}} \subseteq$ \\
\hline 39 & & & & $\mathrm{hB}^{\mathrm{i}} \leftrightarrow \mathrm{hBe}^{\mathrm{i}}$ \\
\hline 40 & & & & $\mathrm{~B}^{\mathrm{i} \leftrightarrow \mathrm{Be}^{\mathrm{i}}}$ \\
\hline 41 & & & Highlighting of the & $\mathrm{hB}^{\mathrm{i}} \leftrightarrow \mathrm{hBe}^{\mathrm{i}}$ \\
\hline 42 & & & $\begin{array}{l}\text { differences between the } \\
\text { expected user and product } \\
\text { behaviours and the real ones }\end{array}$ & $\mathrm{B}^{\mathrm{i}} \leftrightarrow \mathrm{Be}^{\mathrm{i}}$ \\
\hline 43 & & $\mathrm{~B}^{\mathrm{i}} \Leftrightarrow \mathrm{Be}^{\mathrm{i}}$ & \multirow{10}{*}{$\begin{array}{l}\text { Definition of new problem } \\
\text { solving and product } \\
\text { structures }\end{array}$} & $\mathrm{hB}^{\mathrm{i}} \subseteq$ \\
\hline 44 & & $\mathrm{~B}^{\mathrm{i}} \rightarrow \mathrm{S}^{\mathrm{i}}$ & & $\mathrm{hB}^{\mathrm{i}} \Leftrightarrow \mathrm{hBe}^{\mathrm{i}}$ \\
\hline 45 & & & & $\mathrm{hBe}^{\mathrm{i}} \rightarrow \mathrm{hB}^{\mathrm{e}}$ \\
\hline 46 & & $\mathrm{~S}^{\mathrm{i}} \Leftrightarrow \mathrm{Se}^{\mathrm{i}}$ & & $\mathrm{hB}^{\mathrm{i}} \rightarrow \mathrm{B}^{\mathrm{i}}$ \\
\hline 47 & & $\mathrm{Be}^{\mathrm{i}} \rightarrow \mathrm{Se}^{\mathrm{i}}$ & & $\mathrm{B}^{\mathrm{i}} \subseteq$ \\
\hline 48 & \multirow{21}{*}{$\begin{array}{l}\text { Goal feasibility } \\
\text { evaluation }\end{array}$} & $\mathrm{Se}^{\mathrm{i}} \rightarrow \mathrm{S}^{\mathrm{e}}$ & & $\mathrm{B}^{\mathrm{i}} \rightarrow \mathrm{S}^{\mathrm{i}}$ \\
\hline 49 & & $\mathrm{~S}^{\mathrm{e}} \subseteq \mathrm{S}^{\mathrm{i}}$ & & $\mathrm{S}^{\mathrm{i}} \subseteq$ \\
\hline 50 & & & & $\mathrm{~S}^{\mathrm{i}} \Leftrightarrow \mathrm{Se}^{\mathrm{i}}$ \\
\hline 51 & & $\mathrm{~S}^{\mathrm{i}} \rightarrow \mathrm{A}^{\mathrm{i}}$ & & $\mathrm{Be}^{\mathrm{i}} \rightarrow \mathrm{Se}^{\mathrm{i}}$ \\
\hline 52 & & $\mathrm{~A}^{\mathrm{i}} \subseteq$ & & $\mathrm{Se}^{\mathrm{i}} \rightarrow \mathrm{S}^{\mathrm{e}}$ \\
\hline 53 & & $\mathrm{~S}^{\mathrm{i}} \rightarrow \mathrm{M}^{\mathrm{i}}$ & & \\
\hline 54 & & $\mathrm{~A}^{\mathrm{i}} \subseteq \mathrm{M}^{\mathrm{i}}$ & & \\
\hline 55 & & $M^{i} \subseteq$ & & \\
\hline 56 & & $\mathrm{~S}^{\mathrm{i}} \rightarrow \mathrm{E}^{\mathrm{i}}$ & & \\
\hline 57 & & $\mathrm{~A}^{\mathrm{i}} \subseteq \mathrm{E}^{\mathrm{i}}$ & & \\
\hline 58 & & $\mathrm{M}^{\mathrm{i}} \subseteq \mathrm{E}^{\mathrm{i}}$ & & \\
\hline 59 & & $\mathrm{E}^{\mathrm{i}} \subseteq$ & & \\
\hline 60 & & $\mathrm{M}^{\mathrm{i}} \Leftrightarrow \mathrm{Me}^{\mathrm{i}}$ & & \\
\hline 61 & & $\mathrm{E}^{\mathrm{i}} \Leftrightarrow \mathrm{Ee}^{\mathrm{i}}$ & & \\
\hline 62 & & $\mathrm{Me}^{\mathrm{i}} \rightarrow \mathrm{hBe}^{\mathrm{i}}$ & & \\
\hline 63 & & $\mathrm{Ee}^{\mathrm{i}} \rightarrow \mathrm{hBe}^{\mathrm{i}}$ & & \\
\hline 64 & & $\mathrm{hBe}^{\mathrm{i}} \rightarrow \mathrm{hB}^{\mathrm{e}}$ & & \\
\hline 65 & & $\mathrm{hB}^{\mathrm{e}} \subseteq \mathrm{hB}^{\mathrm{i}}$ & & \\
\hline 66 & & $\mathrm{hB}^{\mathrm{i}} \subseteq$ & & \\
\hline 67 & & $\mathrm{hB}^{\mathrm{i}} \rightarrow \mathrm{B}^{\mathrm{i}}$ & & \\
\hline 68 & & $\mathrm{~B}^{\mathrm{i}} \subseteq$ & & \\
\hline
\end{tabular}




\begin{tabular}{|c|c|c|}
\hline $\begin{array}{l}69 \\
70 \\
71\end{array}$ & & $\begin{array}{l}\mathrm{B}^{\mathrm{i}} \Leftrightarrow \mathrm{Be}^{\mathrm{i}} \\
\mathrm{B}^{\mathrm{i}} \rightarrow \mathrm{S}^{\mathrm{i}} \\
\mathrm{S}^{\mathrm{i}} \subseteq\end{array}$ \\
\hline $\begin{array}{l}72 \\
73 \\
74\end{array}$ & $\begin{array}{l}\text { Prototype } \\
\text { generation }\end{array}$ & $\begin{array}{l}\mathrm{S}^{\mathrm{i} \Leftrightarrow \mathrm{Se}^{\mathrm{i}}} \\
\quad \mathrm{Be}^{\mathrm{i}} \rightarrow \mathrm{Se}^{\mathrm{i}} \\
\mathrm{Se}^{\mathrm{i}} \rightarrow \mathrm{S}^{\mathrm{e}}\end{array}$ \\
\hline
\end{tabular}

\subsection{Second $\mathrm{XfD}$ adoption in the field}

The second adoption in the field involves a company producing bathroom fixtures. The company focuses its attention on one side on regulations and standards about health and safety; from the other side, the focus is on UX matters in order to get the best user-product interaction. The redesign activities considered here starts from the analysis of existing products and ends with the development of new releases of them. As for the previous adoption, only the first four phases are considered, up to the highlighting of the differences between the expected users' problem solving and product structures and the real ones (the design activities continue by redesigning the product according to the outcomes of this comparison). The first phase defines the user and product behaviors that designers believe future users expect. This happens considering the functions that the current product makes available. In the second phase, designers interact with the product to infer user and product real behaviors. In this phase, designers analyze the structures of the product to infer its behaviors. The third phase highlights the differences between the user and product expected behaviors and the real ones, allowed by the current product. The fourth phase defines new problem solving and product structures according to the results of the previous one.

Four designers of washbasins were involved. They applied the XfD and generated the model shown in tab. 1, right side. The generation of the model took one hour and forty minutes; the designers needed thirty-five minutes to apply the statements, forty-five minutes to apply the rules and twenty minutes to implement the suggested subprocesses. Designers needed four times the help of the XfD developers in applying the rules. Specifically, it happened in the first and in the second phases, for introducing the meaning variable and the related sub-processes, with the same reason as in the first adoption. In the fourth phase, designers needed help for introducing the transformation between the interpreted human behaviors and the interpreted product behaviors because they presumed that this had been already considered at the end of the second phase. Always in the four phase, designers needed help for introducing the relationships between the expected behaviors and the expected structures of the product because they presumed that this had already been considered at the beginning of the second phase.

Once generated the model, all the designers filled the first questionnaire. After that, two designers performed the original activities and the other two the enhanced ones, all of them aiming at redesigning a washbasin with an automatic faucet and a soap dispenser. As the previous adoption, at the end the designers who performed the enhanced activities filled the second questionnaire.

It is worth to say that, in this adoption, the design outcomes refer to product structures (S) (as in the first adoption) and to problem solving processes, translated into human behaviors (hB). Until now, hB has been just an internal variable of the XfD. On the contrary, this adoption makes clear that hB must become part of the sources/targets set and considered like the product behavior variable $\mathrm{B}$. The $\mathrm{XfD}$ architecture has been modified accordingly.

\subsection{Analysis of the results}

Tab. 2 shows the quantification of the data collected by the $\mathrm{XfD}$ developers during the executions of the original and of the enhanced design activities in the two adoptions. These data consist of the number of design outcomes generated and the time spent to perform the activities. The second company had two different types of design outcomes (problem solving processes and product structures); the data have been classified accordingly.

Table 2. Data collected during the executions of the original and of the enhanced design activities.

\begin{tabular}{|c|c|c|c|c|}
\hline \multirow[b]{2}{*}{ Activities } & \multicolumn{2}{|c|}{$\begin{array}{l}\text { First adoption } \\
\text { (chair design) }\end{array}$} & \multicolumn{2}{|c|}{$\begin{array}{l}\text { Second adoption } \\
\text { (washbasin } \\
\text { redesign) }\end{array}$} \\
\hline & Original & Enhanced & Original & Enhanced \\
\hline $\begin{array}{l}\text { Design } \\
\text { outcomes } \\
(\mathrm{hB})(\#)\end{array}$ & N/A & N/A & 4 & 7 \\
\hline $\begin{array}{l}\text { Design } \\
\text { outcomes }(\mathrm{S}) \\
(\#)\end{array}$ & 21 & 33 & 11 & 21 \\
\hline Time (min) & 220 & 245 & 105 & 118 \\
\hline
\end{tabular}

The enhanced activities always generated more design outcomes than the original ones (in the second adoption, the design outcomes are almost twice). Regarding the time spent to perform the activities, the enhanced ones always took longer than the original ones; the difference is around $12 \%$. At this stage of the research, it is not possible to evaluate the real impact of this difference. Nevertheless, as inferred from the second questionnaire, designers recognized the improvements in the design outcomes from the quantitative and qualitative points of view and accepted the longer time as the right price to pay.

From the qualitative point of view, most of the design outcomes defined by the original activities appear also in the enhanced ones. Nevertheless, the enhanced activities generated further design outcomes that could innovate the product.

Considering the first adoption, two design outcomes present in both the original and enhanced activities are "the chair should have an ergonomic and padded seat, pivoting in order to facilitate standing up without moving the chair from the table" and "the armrests should be adjustable respect to the users' height". Regarding the design outcomes generated thanks to the enhancement, the rules suggested, for example, considering meanings during the goal definition. This corresponded to the addition of the subprocesses $\mathrm{F}^{\mathrm{i}} \rightarrow \mathrm{M}^{\mathrm{i}}, \mathrm{M}^{\mathrm{i}} \Leftrightarrow \mathrm{Me}^{\mathrm{i}}$ and $\mathrm{Me}^{\mathrm{i}} \rightarrow \mathrm{hBe}^{\mathrm{i}}$ and allowed generating five new design outcomes. Regarding one of 
these, the designers thought about the meaning "cook" and, from this, generated the new design outcome "the chair should allow modifying the height by pulling a lever in order to make the user able to reach very high sideboards to get ingredients without standing up and by pushing the same lever to return in the previous position". This design outcome enhances the functions of the chair by adding the possibility to reach heights impossible for similar products; at the same time, the different movements of the lever (pull and push) avoid performing the same action to get opposite results, classic example of contradiction that can reduce the usability of the product. The rules suggested enhancements also about considering the generation of prototype structures starting from product behavior $\left(\mathrm{Be}^{\mathrm{i}} \rightarrow \mathrm{Se}^{\mathrm{i}}\right)$ and this led to four new design outcomes. One of them is "the chair armrests can be locked at the lowest position, aligned with the seat, to be used as rail to guide and fix carrycots". This design outcome enhances the safety of the product use by exploiting the armrests as fixed support board without the need to fix carrycots using different tools.

The second adoption shows common design outcomes between the execution of the original and enhanced design activities as well. For example, both the groups of designers identified the design outcomes "there is the need of a finer regulation system of the water temperature" and "the space range to activate the faucet with the hands must be increased respect to the users' heights and arm lengths". Contrarily, only the designers performing the enhanced activities found further design outcomes that could innovate and make the product more useful. For example, the rule suggesting the consideration of the influence of meanings on human behaviors $\left(\mathrm{Me}^{\mathrm{i}} \rightarrow \mathrm{hBe}^{\mathrm{i}}\right)$ allowed generating four new design outcomes. One of them is "a led should be placed on the faucet to inform the users if the faucet is idle but ready (green), running (red) or unavailable (switched off)". This design outcome enhances product affordance because it shows in advance the state of the product to the users, without requiring any action to know it. Another rule suggested considering human behaviors in generating product behaviors $\left(\mathrm{hB}^{\mathrm{i}} \rightarrow \mathrm{B}^{\mathrm{i}}\right)$. This allowed defining two new design outcomes; one of them is "a footstool should be embed in the washbasin in order to allow any user approaching the faucet correctly and activating the water". This design outcome enhances the accessibility of the product to every user, including those having particular physical characteristics or disabilities.

Regarding usability concerns, the first questionnaires filled by the designers reported that the statements were difficult to apply at the beginning because getting inside the modeling logic was not straightforward. Once overcome these initial difficulties, the statements became quite easy to apply. Designers met more difficulties in understanding and applying the rules than the statements; this happened because the rule adoption requires deeper knowledge about cognitive processes and emotional matters than the application of the statements.

\section{Discussion}

The first XfD adoptions in the field start demonstrating its value and highlighting its compliance with the requirements set at the beginning. Regarding REQ1, the set of sources/targets has been implemented and appears as correctly managed since the pieces of information that these variables represent are elaborated using sub-processes built by obeying to the FBS. About REQ2, users are correctly kept into consideration thanks to the generation and management of specific variables related to product experience and human behavior. These variables have a great importance because their analysis is the starting point for defining the expected behaviors of products; moreover, they are the first expected variables defined in all the design activities considered here; the values of the other variables and consequently of the targets are always computed starting from them. For what concerns REQ3, the XfD architecture is quite easy to modify and it seems flexible enough to adapt to heterogeneous application contexts in the product development domain (e.g., the second adoption in the field required the variable $\mathrm{hB}$ to become an element of the sources/targets set rather than an internal variable); moreover, if design activities should change over time, the XfD model can be easily updated in an incremental way, without the need to restart from the beginning. Regarding REQ4, the XfD tools seem to support researchers and/or designers in the modeling in a usable way. The positive feedback emerged from the first questionnaires filled by the designers demonstrates this. Designers who performed the enhanced design activities reputed the enhancements important and useful because they introduced a more ordered ways to conduct the activities and to achieve new design outcomes. Moreover, the rules proved to be able to improve the quality of these outcomes as reported by the designers in the second questionnaire. The new design outcomes would enhance the capability of avoiding contradictions in defining different movements of the chair lever in the first adoption as well as the accessibility and affordance of the washbasin in the second adoption.

It is also worth to say that the XfD development highlighted once more the tight relationship between the selection of the sources/targets and the development of the sub-processes to manage them. This relationship allows a sort of "reverse design" concept to be introduced. Available information/knowledge about specific design activities, along with their temporal sequence, could optimize the selection of the right input to consider (sources) as well as of the best points of view for representing the output (targets). For example, consider a company developing user interfaces for office automation software packages. Current design activities could likely focus mainly on the interface structure (buttons, icons, widgets in general); therefore, the target is the product structure. The XfD-based analysis would highlight activities allowing a better understanding of those human behaviors aimed at satisfying users' specific needs. Consequently, the product behavior should be designed and developed in order to offer the best support in terms of cognitive compatibility. All of this would make the interface structure (previous goal of the design activities) relegated to the mere implementation of the much more important definition of the product behavior (new goal of the design activities).

The current state of the research shows also some drawbacks. The first adoptions of the XfD in the field are not enough to say the ultimate word about its value. Moreover, no comparisons with other descriptive methods and tools different from the FBS have been performed yet. Statements and rules are not fully developed; this could make the use of the XfD by generic practitioners not expert in cognitive and user experience matters quite hard without help. This is demonstrated by the required interventions of the developers during the first two adoptions. Moreover, the time required to apply the statements and rules is the 
symptom of the lack of automatisms in the XfD. Another drawback comes from the difficulties found by some XfD users in understanding the actual advantages brought by the enhanced design activities. For example, the first company evaluated negatively the addition of the transformation of behaviors into structures $\left(\mathrm{Be}^{\mathrm{i}} \rightarrow \mathrm{Se}^{\mathrm{i}}\right.$, sub-process \#73 in tab. 1 , left side) in the prototype generation phase. Designers were quite skeptical about moving this activity from the evaluation of the prototype (phase not considered here) to the prototype generation as suggested by the model. To them, this shift would generate a waste of time because they were convinced that they did not achieve any further design outcome so early. One more drawback focuses on the sources/targets set. It includes only the FBS variables; it neglects the new variables aesthetics, meaning and emotion. This means that, although REQ1 is satisfied quite well thanks to the exploitation of the FBS variable $\mathrm{S}$ (it can represent the product from different points of view like structure, materials, sounds, etc.), other important elements like emotions, meanings, etc., cannot be used as pieces of information where the design activities start from and/or as expected results. This would also happen for variables related to human personality, environment characteristics, etc., not considered now. Finally, the application domains have been intentionally restricted to the sole product development in order to study such kind of design activities and develop this first release of the XfD as best as possible; as a consequence, it is impossible to guarantee the $\mathrm{XfD}$ value for different application domains than this (e.g., services generation, business and marketing analyses, etc.).

\section{Conclusions}

Starting from well-known existing methods and tools focused on modeling design activities, on human cognition and emotions and from real experiences in the field, the research described in this paper proposed the $\mathrm{X}$ for Design (XfD) framework. It allows modeling design activities dealing with different starting points and goals as well as with user experience concerns like emotions and human behaviors. The goal is to enhance these activities by highlighting where to add something new and/or what to modify/delete of the existing. The validation of the XfD has started with two adoptions in the field; they highlighted the capability of the XfD to overcome the weaknesses of existing modeling methods and tools. Specifically, the XfD can consider different sources, like product functions, shapes or behaviors, and can model activities that aim at developing targets focused on different product aspects, like functions, behaviors, etc. Moreover, the XfD considers several aspects of the user experience together and gives them a precise role in the framework, as witnessed by the presence of the A, M, E and hB variables. Finally, the XfD can enhance the design activities in order to increase the number of design outcomes as well as their quality and innovation degree. Thanks to all of this, the XfD could help researchers in deepening their knowledge about the role of emotions and human behaviors in design; at the same time, designers could adopt the framework to enhance their activities in order to match current market requirements at best.

In the near future, other adoptions in the field and other existing descriptive methods and tools will be considered in order to validate the XfD and update/integrate it, focusing mainly on the development of full sets of statements to translate the design activities and of rules to refine the resulting models. The introduction of some automatisms will speed up the XfD adoption. Among them, relationships between statements and rules will allow knowing which rules could be involved in enhancing specific design activities respect to the used statements and helps in implementing the refined sub-processes into design activities will explain the possible advantages of their exploitation. All of this will lead towards a further improvement of the XfD usability for practitioners not expert in cognitive and user experience matters. Another important hint for future work refers to the sources/targets set. The A, M and E variables, currently internal variables, will be moved into this set because some design activities could need to start from them as well as consider them as targets. Moreover, the set content could be integrated by introducing variables related to human personality and environment characteristics. Some more reasoning on the meaning of all the variables is needed to understand and decide if the FBS variables $\mathrm{N}$ and $\mathrm{R}$ should be represented by the $\mathrm{A}, \mathrm{M}, \mathrm{E}$ and $\mathrm{hB}$ variables in order to reduce the complexity of the XfD architecture. The current restriction on the application domains (corresponding to the sole product development) will be overcome by adding new requirements, statements and rules in order to make the framework able to model even more heterogeneous design activities. Finally, future work will also focus on the "reverse design" concept; its possible exploitation has just been spotted in the discussion section but it deserves some more reasoning.

This is an Open Access article distributed under the terms of the Creative Commons Attribution License

\section{References}

1. X. Y. Li and M. Gunal, "Exploring cognitive modelling in engineering usability design", Journal of Engineering Design, 23(1-3), pp. 77-97 (2012).

2. J. Yoon, A. E. Pohlmeyer and P. M. A. Desmet, "When 'Feeling Good' is not Good Enough: Seven Key Opportunities for Emotional Granularity in Product Development", International Journal of Design, 10(3), pp. 1-15 (2016).

3. P. M. A. Desmet and P. Hekkert, "Framework of product experience", International Journal of Design, 1(1), pp. 57-66 (2007).

4. M. Winckler, R. Bernhaupt and C. Bach, "Identification of UX dimensions for incident reporting systems with mobile applications in urban contexts: a longitudinal study", Cognition, Technology and Work, 18, pp. 673-694 (2016).

5. S. Filippi and D. Barattin, "Extending the situated functionbehaviour-structure framework to human-machine interaction", International Journal of Interactive Design and Manufacturing, 11(2), pp. 247-261 (2017).

6. X. Li, "Quantitative Research on Product Form Based on Kansei Engineering", Journal of Engineering Science and Technology Review, 11 (1), pp. 84-89 (2018).

7. Y. Bi, S. Li, D. Wagner and T. Reid, "The impact of vehicle silhouettes on perceptions of car environmental friendliness and 
safety in 2009 and 2016: a comparative study", Design Science Journal, 3, pp. 1-33 (2017).

8. M. Ashby and K. Johnson, "Materials and Design. The art and science of material selection in product design". ButterworthHeinemann Elsevier (2014).

9. Y. Takada, E. R. Boer and T. Sawaragi, "Driver assist system for human-machine interaction", Cognition, Technology and Work, 19, pp. 819-836 (2017).

10. S. Graziosi, F. Ferrise, G. P. Furtado and M. Bordegoni, "Reverse engineering of interactive mechanical interfaces for product experience design", Virtual and Physical Prototyping, 9(2), pp. 65-79 (2014).

11. J. S. Gero and U. Kannengiesser,"The situated functionbehaviour-structure framework", Design Studies, 25(4), pp. 373391 (2004).

12. I. Horvath, "A treatise on order in engineering design research", Research in Engineering Design, 15(3), pp. 155-181 (2004).

13. S. A. Gregory, "The Design Method". Springer (2013).

14. N. Cross, "Engineering Design Methods (4rd ed.)". Wiley (2008).

15. G. Pahl, W. Beitz, J. Feldhusen and K. H. Grote, "Engineering Design - A Systematic Approach", Third Edition. SpringerVerlag (2007).

16. U. Kannengiesser and J. S. Gero, "Can Pahl and Beitz' systematic approach be a predictive model of designing?", Design Science Journal, 3, pp. 1-20 (2017).

17. G. Q. Huang and K. L. Mak, "Developing a generic design for X shell", Journal of Engineering Design, 8(3), pp. 251-260 (1997).

18. R. Holt and C. Barnes, "Towards an integrated approach to "design for X": An agenda for decision-based DFX research", Research in Engineering Design, 21(2), pp. 123-136 (2010).

19. T. Z. Chen and Y. M. Mo, "Influence of strength grade and surface finish on threaded fastener for reliability application", Journal of Engineering Science and Technology Review, 6 (5), pp. 95-100 (2013).

20. J. S. Gero, "Design prototypes: a knowledge representation schema for design", AI Magazine, 11(4), pp. 26-36 (1990).

21. J. S. Gero, H. Jiang and C. B. Williams, "Design cognition differences when using unstructured, partially structured and structured concept generation creativity techniques", International Journal of Design Creativity and Innovation, 1(4), pp. 196-214 (2013).
22. K. Dorst and P. E. Vermaas, "John Gero's Function-BehaviourStructure model of designing: a critical analysis", Research in Engineering Design, 16, pp. 17-26 (2005).

23. P. Galle, "The ontology of Gero's FBS model of designing", Design Studies, 30, pp. 321-339 (2009).

24. B. Helms, K. Shea and F. Hoisl, "A framework for computational design synthesis based on graph-grammars and functionbehaviour-structure", In the International Design Engineering Technical Conference \& Computers and Information in Engineering Conference, IDETC/CIE, pp. 841-851 (2009).

25. G. Cascini, G. Fantoni and F. Montagna, "Situating needs and requirements in the FBS framework", Design Studies, 34(5), pp. 363-662 (2012).

26. M. Hassenzahl and N. Tractinsky, "User experience - a research agenda", Behaviour \& Information Technology, 25(2), pp. 91-97 (2006).

27. P. Jordan, "Designing pleasurable products: an introduction to the new human factors". Taylor \& Francis (2000).

28. D. Norman, "The design of everyday things, Revised and expanded edition". Basic Book (2013).

29. J. Astoul, J. Geneix, E. Mermoz and M. Sartor, "A simple and robust method for spiral bevel gear generation and tooth contact analysis", International Journal of Interactive Design and Manufacturing, 7(1), pp. 37-49 (2013).

30. C. Brecher, P. Utsch, R. Klar and C. Wenzel, "Compact design for high precision machine tools", International Journal of Machine Tools and Manufacture, 50(4), pp. 328-334 (2010).

31. A. Alessi,"The dream factory: Alessi since 1921". Electa/Alessi (1998).

32. S. Filippi and D. Barattin, "Definition of the form-based design approach and description of it using the FBS framework", In the International Conference on Engineering Design - ICED 2015 (eds. C. Weber, S. Husung, M. Cantamessa, G. Cascini, D. Marjanovic and S. Venkataraman), pp. 65-76 (2015).

33. H. Tamura, H. Yamamoto and A. Katayama, "Mixed reality: future dreams seen at the border between real and virtual worlds", IEEE Computer Graphics and Applications, 21(6), pp. 64-70 (2001).

34. D. G. Ullman, "The mechanical design process. Fourth edition". McGraw-Hill (2010). 